\title{
ARTICLE \\ Radiation Shielding Program BULK-II for Proton and Carbon Accelerator Facilities
}

\author{
Hiroshi IWASE $^{1, *}$, Ryuichi TAYAMA ${ }^{2}$, Tomoharu HASHIMOTO ${ }^{2}$, Yoshihito NAMITO ${ }^{1}$, and Syuichi BAN ${ }^{1}$ \\ ${ }^{1}$ High energy accelerator research organization (KEK), Oho 1, Tsukuba 305-0801, Japan \\ ${ }^{2}$ Hitachi-GE Nuclear Energy, Sawai 3-1-1, Hitachi 317-0073, Japan
}

\begin{abstract}
A simple but reliable radiation shielding program BULK-II wad developed for proton and carbon accelerator facilities at incident energies of $50-400 \mathrm{MeV} /$ nucleon. Particle-therapy facility design is the main target of BULK-II. Neutron and gamma doses can be calculated behind iron, concrete, or both shielding materials. For beam stopping target either carbon or iron is available. The basic formula is the Moyer formula and the build-up effect is also included.

In order to verify the accuracy of BULK-II, benchmark calculations of the angular distribution without shielding and deep penetration in concrete shielding of the dose of ${ }^{12} \mathrm{C}$ induced secondary neutrons were performed and compared with measured data. The agreement is reasonable and the reliability of BULK-II is confirmed. The BULK-II program is open to the public and can be obtained at http://rcwww.kek.jp/BULK-II.
\end{abstract}

KEYWORDS: neutron dose, proton therapy, carbon therapy, shielding design

\section{Introduction}

In particle accelerator facilities fast neutrons are produced by the nuclear reaction of projectiles with accelerator components. High-energy neutrons possibly travel long distances penetrating through shielding materials and causing radiation dose to improper objects - like humans. The neutron dose is the main purpose for radiation shielding and therefore an accurate estimation of the neutron dose is essential for the shielding design of particle accelerators. The medical use of particle accelerators becomes very common and the number of particle therapy facilities is increasing. A simple program for shielding design is really required. Therefore we developed a radiation shielding program, BULK-II, for proton and carbon accelerator facilities in the incident energy region of 50 - $400 \mathrm{MeV} /$ nucleon.

\section{Concept and Equations}

\section{Moyer model}

The Moyer formula ${ }^{1)}$ is widely used for radiation protection purposes. The concept of Moyer model is based on the spectrum equilibrium, i.e., when neutrons travel deep into shielding material (deep penetration), the distribution of neutron energies does not change for any different thickness of shielding (called spectrum equilibrium). The spectrum equilibrium can be seen for any materials at positions deeper than a certain thickness, and since the energy distribution does not change, neutron attenuation can be written simply independent to neutron energy and represented by only one parameter, "attenuation length $\lambda$ " (otherwise attenuation length should be prepared for various energy bins). The Moyer formula is shown in Eq.(1).

${ }^{*}$ Corresponding author, Phone:+81-(0)29-879-6004, Fax:+81-(0)29-8644051, and E-mail: hiroshi.iwase@kek.jp

(c)Atomic Energy Society of Japan

$$
H(r, t)=H_{0} \cdot \frac{1}{r^{2}} \cdot e^{-\frac{t}{\lambda}}
$$

, where $H_{0}$ is a dose as a source term, $\mathrm{r}$ is a distance from the source to a shielding calculation position, $t$ is a shileing penetration thickness, as seen in Fig. 1.

The attenuation can be calculated by an exponential equation using an attenuation length $\lambda$.

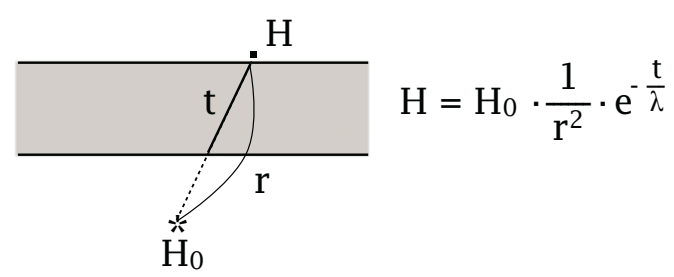

Fig. 1 Moyer model of radiation shielding calculation

\section{Build-up effect}

Neutron dose penetration can be described by the Moyer formula under the condition of spectrum equilibrium. On the other hand, in the early stage of the penetration, the "Buildup effect" occurs where the number of scatterd neutrons increases and the distribution of neutron energies also changes through shielding material. The build-up effect continues to a certain thickness, then gradually disappears and spectrum equilibrium is reached. Figure. 2 shows the typical neutron attenuation in matter; comparing (1) a simple equilibrium condition case and (2) a case which includes a build-up effect. The gradients (attenuation length $\lambda$ ) are the same in both cases, but the resulting doses at the same shielding thickness disagree due to the build-up effect.

\section{Concept of the BULK-I program}

In high-energy accelerator facilities, including the build-up effect is the key issue to perform accurate shielding design 


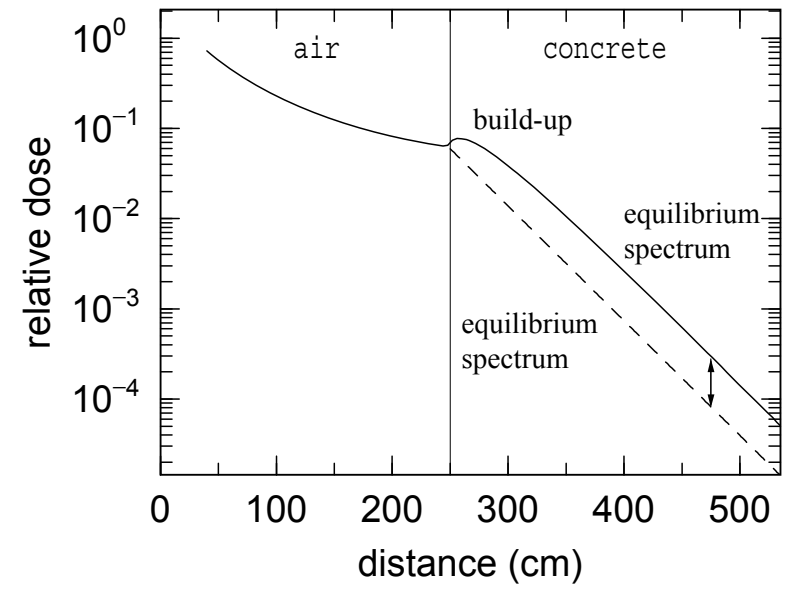

Fig. 2 Conceptual plot of neutron dose attenuation between an equilibrium spectrum case and a case which includes a buildup effect. The solid line is a PHITS calculation of $100 \mathrm{MeV}$ mono-energetic and isotropic neutrons in a concrete shield $\left(\rho=2.3 \mathrm{~g} / \mathrm{cm}^{3}\right)$.

using the Moyer type simple formula. The BULK-I code was developed by Tayama et al. ${ }^{2)}$ for proton accelerator facility by including the build-up effect to the Moyer formula, as shown in Eq.(2),

$$
H(r, t)=H_{0} \cdot \frac{1}{r^{2}} \cdot e^{-\frac{t}{\lambda}}\left\{\alpha-\left(1-e^{-\beta t}\right) \cdot(\alpha-1)\right\} .
$$

The first part is identical to the Moyer model, but a build-up effect correction term in brace \{\} is included in the equation, where $\alpha$ is the ratio of $H(r, 0) r^{2}$ to $H_{0}$, and $\beta$ a fitting parameter of the build-up correction.

The parameters in Eq. $(2)\left(H_{0}, \lambda, \alpha\right.$, and $\left.\beta\right)$ are calculated by MCNPX $2.1 .5^{3)}$. Figure 3 shows the calculation geometry for the parameter estimation. For 12 angular regions of 0 to 180 degrees, three energy regions $(<0.414 \mathrm{eV}, 0.414 \mathrm{eV}-5$ $\mathrm{MeV}, \geq 5 \mathrm{MeV})$ and four iron shielding thickness (0, 25, 50, $70 \mathrm{~cm}$ ) plus $400 \mathrm{~cm}$ thick concrete shielding, doses are calculated using MCNPX 2.1.5. In each calculation, the parameters $H_{0}, \lambda, \alpha$, and $\beta$ are obtained.

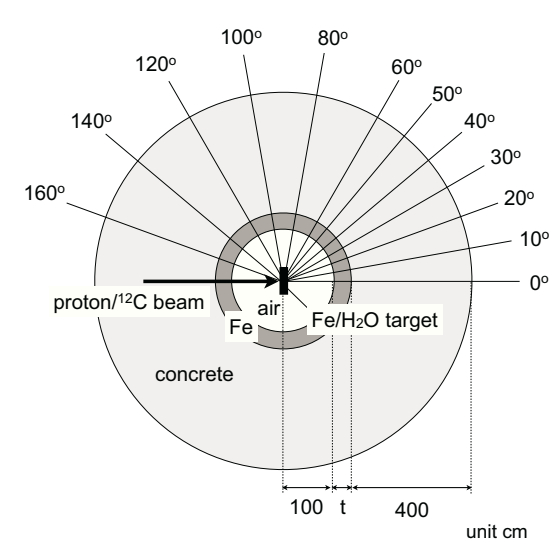

Fig. 3 Geometry for calculation of parameters $H_{0}, \lambda, \alpha$, and $\beta$ used in BULK-I/BULK-II codes

\section{Dose conversion factors}

Fluence to effective dose conversion factors used in BULKII are based on ICRP publ.74 ${ }^{4}$ for photons and neutrons up to $200 \mathrm{MeV}$, and on the evaluated data by Iwai et al. ${ }^{5)}$ for neutrons above $200 \mathrm{MeV}$. The neutron dose conversion factors are shown in Fig.4.

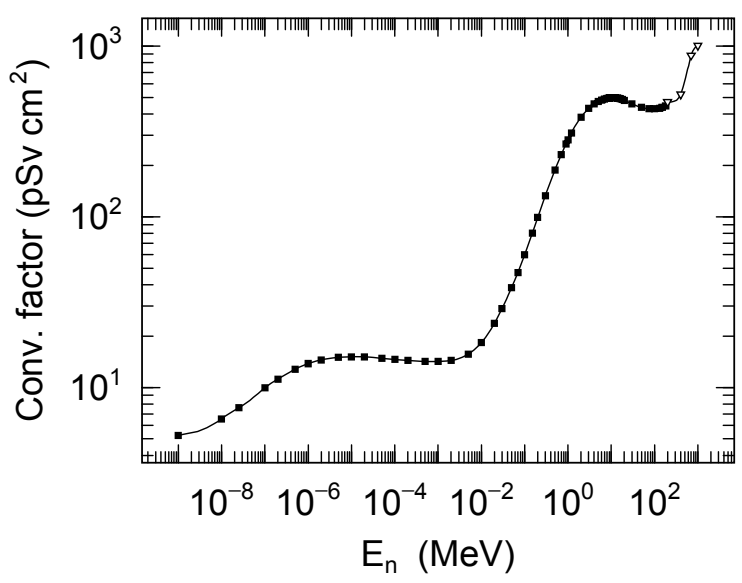

Fig. 4 Neutron dose conversion factors for different neutron energies by ICRP 74 (below $200 \mathrm{MeV}$, square) and by Iwai et al. ${ }^{5)}$ (above $200 \mathrm{MeV}$, triangle).

\section{Development of BULK-II (Extension to ${ }^{12} \mathrm{C}$ ac- celerator facility)}

In order to calculate ${ }^{12} \mathrm{C}$ induced neutron dose for shielding design for ${ }^{12} \mathrm{C}$ accelerator facilities such as carbon therapy, BULK-I was upgraded to BULK-II in this study. The basic concepts and equations are the same between BULK-I and BULK-II, but the parameter sets of $H_{0}, \lambda, \alpha$, and $\beta$ for ${ }^{12} \mathrm{C}$ induced reactions were changed from those for proton-induced reactions in BULK-I. For the parameter calculations, ${ }^{12} \mathrm{C}$ reactions $\left(400,300,250,200,150\right.$, and $100 \mathrm{MeV} /$ nucleon ${ }^{12} \mathrm{C}$ ions on water and iron targets which are a few $\mathrm{cm}$ thicker than the $\mathrm{C}$ ion ranges) were calculated by the PHITS code ${ }^{6)}$ and then the produced neutrons were inputted to MCNPX 2.5.0 using the geometry shown in Fig.3. The obtained parameters are listed in a BULK-II manual at http://rcwww.kek.jp/BULK-II/. In the ${ }^{12} \mathrm{C}$ induced reactions, not only neutrons but many protons and ${ }^{4} \mathrm{He}$ ions are produced as fragments of incident ${ }^{12} \mathrm{C}$ at a forward angle. The amounts of ${ }^{4} \mathrm{He}$ ions produced are twice than those of neutrons and protons around 0 degrees ${ }^{7}$. However the produced protons and ${ }^{4} \mathrm{He}$ ions are not included in BULK-II. In a recent estimation by PHITS, the ions contribute about $30 \%$ of the neutron dose on the beam axis. The contribution of such secondary charged particle dose to neutron dose shall be much more accurately estimated and included as additional correction factors to be multiplied to the final result of BULK-II. 


\section{Benchmark calculations}

\section{Angular distribution of ${ }^{12} \mathrm{C}$ neutron dose: source term}

Neutron dose just behind a beam-stopping target has been calculated by BULK-II and compared with the experimental data measured at GSI (Gesellschaft für Schwerionenforschung) Germany ${ }^{8)}$. The experiment was performed as follows: $200 \mathrm{MeV} /$ nucleon ${ }^{12} \mathrm{C}$ ions were stopped in a $12.8 \mathrm{~cm}$ water target, a WENDI ${ }^{9)}$ wide energy-range neutron dosimeter was placed $300 \mathrm{~cm}$ away from the target, and the neutron dose was measured with changing detection positions. The position was changed to have detection angles of $0,5,10,20$, $30,60,90$ and 135 degrees in order to obtain an angular distribution of the neutron dose. The results are shown in Fig.5. The comparison shows that reasonable agreement is obtained between GSI data and the calculation by BULK-II. Some discrepancies can be seen especially at 10 and 20 degrees, and the same tendency was obtained in the previous comparison ${ }^{7)}$ between measured neutron energy spectra ${ }^{7)}$ and PHITS calculations.

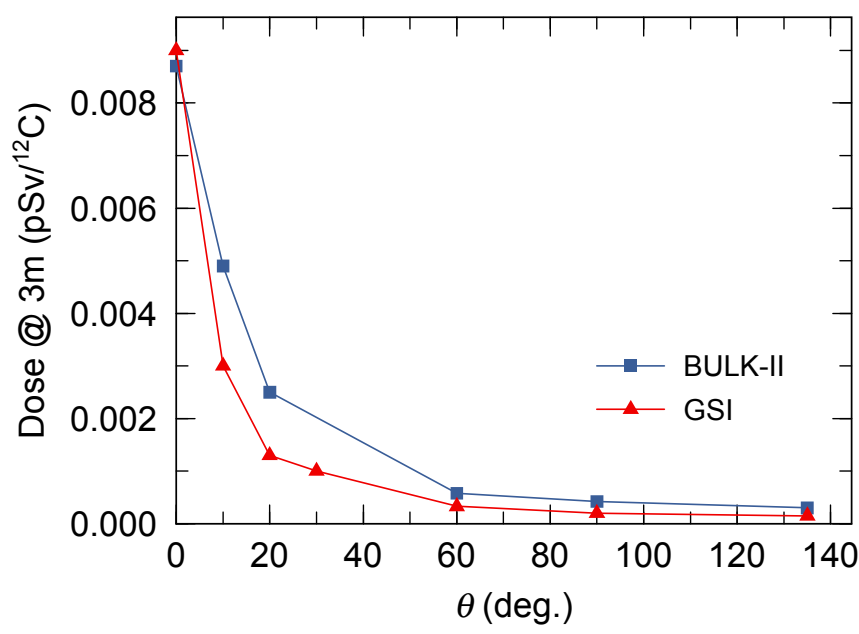

Fig. 5 Comparison of BULK-II calculation and measured GSI data on angular distribution of neutron dose from $12.8 \mathrm{~cm}$ thick water bombarded by $200 \mathrm{MeV} /$ nucleon ${ }^{12} \mathrm{C}$

It is noted that neutron doses calculated in Fig. 5 are not in or behind shielding wall but just in front of the shielding and then calculated results at dose calculating positions include backscattered neutron contribution from the shielding. In order to estimate and subtract the contribution of the backscattered neutron, additional PHITS calculations were performed; neutron doses behind the target were calculated with and without the shielding wall for all detection angles. The ratio of both results is the contribution of backscattered neutrons. The estimated backscattered neutron contribution factors (neutron dose with shield /without shield) are; 1.49, 1.46, and 1.42 for 0,10 , and 20 degrees, respectively, and the BULK-II result shown in Fig.5 has already been corrected (reduced) by the ratios.

\section{Deep attenuation calculation of ${ }^{12} \mathrm{C}$ neutron dose}

In order to check the accuracy of BULK-II especially for use of an actual shielding design, a benchmark calculation has been performed by comparing the HIMAC data by Sasaki et $\mathrm{al}^{10)}$. The experimental setup is shown in Fig.6. 400 $\mathrm{MeV} /$ nucleon ${ }^{12} \mathrm{C}$ ions were stopped in a $5 \mathrm{~cm}$ copper target, $50 \mathrm{~cm}$ concrete shielding blocks were placed $126 \mathrm{~cm}$ downstream from the target, and neutrons were detected behind the shielding with a $12.7 \mathrm{~cm}$ diam by $12.7 \mathrm{~cm}$ long NE213 scintillator coupled with an NE102A plastic veto scintillator. The data was taken for concrete thickness of 50, 100, 150, and 200 $\mathrm{cm}$. For each thickness neutron energy spectrum above 20 $\mathrm{MeV}$ was obtained by unfolding the measured NE213 pulseheight distribution ${ }^{10)}$.

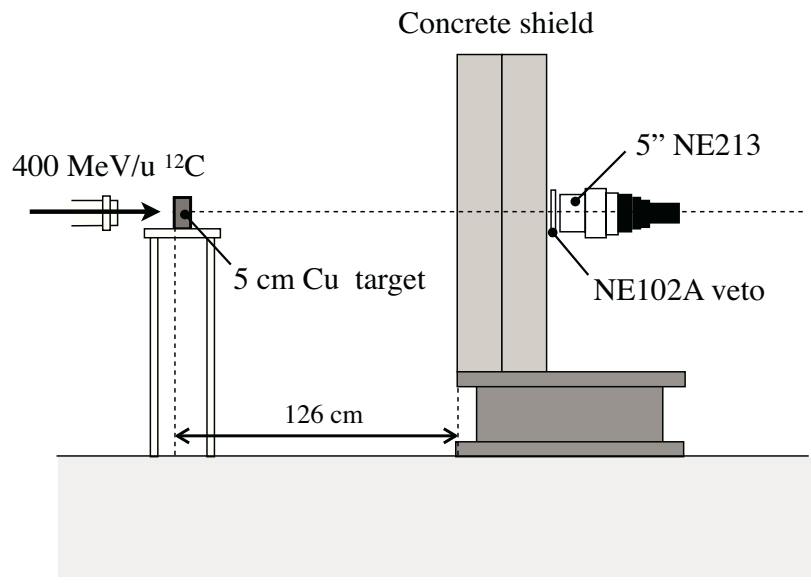

Fig. 6 Experimental setup of Sasaki's HIMAC shielding experiment $^{10)}$

In this work the measured neutron energy spectrum for each concrete thickness was converted to neutron dose by multiplying the conversion factor by Iwai et al. ${ }^{5)}$. BULK-II calculation was performed to estimate neutron doses behind the concrete shield of different thickness. The target material was iron instead of copper in the calculation because only iron and water targets are available in the BULK-II code. A full3D Monte Carlo calculation by PHITS was also performed for comparison.

Figure. 7 shows the compared results. Since the experimental data by Sasaki et al. were obtained above the neutron energy of $20 \mathrm{MeV}$, the doses plotted in Fig.7 are neutron dose above $20 \mathrm{MeV}$. In order to estimate the contribution of neutrons below $20 \mathrm{MeV}$ to the total dose, additional PHITS calculations were performed. Neutron energy spectra behind the different thick concrete shields were calculated. For each energy spectrum, neutron doses with two different threshold energy of 0 (A: total neutron dose ), and $20 \mathrm{MeV}$ (B: neutron dose above $20 \mathrm{MeV}$ ) were calculated. Sasaki's data correspond to the $\mathrm{B}$. The ratio $\mathrm{B} / \mathrm{A}$ is the contribution of neutrons above $20 \mathrm{MeV}$ to the total neutron dose, and the calculated ratios are, $0.74,0.73,0.71$, and 0.76 behind $50,100,150$, and $200 \mathrm{~cm}$ concrete shield respectively. The Sasaki's data were corrected (divided) by the factors and plotted by the dashed 


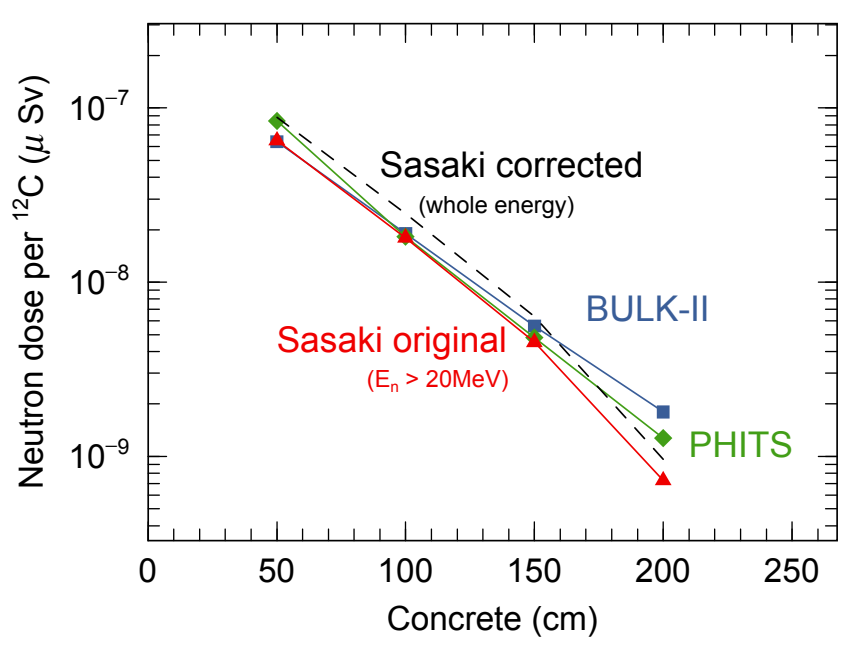

Fig. $7400 \mathrm{MeV} /$ nucleon ${ }^{12} \mathrm{C}$ neutron dose deep penetration in concrete by BULK-II, Sasaki's data ${ }^{10)}$ and PHITS Monte Carlo simulation. The dashed line is a corrected version of Sasaki's data.

line in Fig.7.

The agreement between BULK-II, Sasaki's data, and PHITS is fairly good especially at thin-concrete thicknesses of 50,100, and $150 \mathrm{~cm}$. At $200 \mathrm{~cm}$ concrete thickness they agree within a factor of three. Considering the uncertainties of both experiment and calculation, possibly rough estimation of the concrete density (e.g., lack of Fe frames inside concrete) and components of concrete material in the calculation. It can be said that the discrepancy at $200 \mathrm{~cm}$ is rather acceptable. On the other hand it can also be said that neutron data behind concrete thicker than $200 \mathrm{~cm}$ is also required since typical concrete wall for carbon therapy room comes to $300 \mathrm{~cm}$. Further experimental study should be performed to verify the accuracy of the Monte Carlo codes and BULK-II.

It is worthy of special mention that the results calculated by such the simple formula BULK-II shows practically the similar values calculated by a full Monte Carlo treatment by PHITS. BULK-II is an excel macro program and the use is remarkably simple. Shielding design for particle therapy facility can be easily and very quickly carried out by using BULK-II. The merit of the use of BULK-II is especially that almost no human-induced error can be included to result, and one can obtain an order estimation of neutron dose (with the accuracy shown in this paper) at any shielding conditions in a minute.

\section{Summary and conclusion}

A simple but reliable radiation shielding calculation program for proton and carbon accelerator facilities BULK-II has been developed in this study. The basic concept of BULKII is the same to the previous version of BULK-I, such that the build-up effect, which plays an important role to radiation protection calculation in recent high energy accelerator facilities, included to the Moyer formula. Two benchmark calculations has been done; (1) angular distribution of ${ }^{12} \mathrm{C}$ induced secondary neutron dose (source term) and (2) deep penetration of ${ }^{12} \mathrm{C}$ induced secondary neutron dose in concrete shield. The BULK-II results show reasonable agreement to measured data and give practically the similar result to 3dimensional full Monte Carlo calculation. BULK-II is quite useful tool to perform shielding design of particle therapy facilities. BULK-II is an excel macro program and open to public at http://rcwww.kek.jp/BULK-II.

\section{References}

1) Moyer B. J., "Evaluation of Shielding Required for the Improved Bevatron," Rep. UCRL-9796, aLawrence Berkeley Lab., Berkeley, CA (1961).

2) R. Tayama et al., "Development of a Radiation Shielding Tool for Proton Accelerator Facilities (BULK-I)," SATIF7 (2004).

3) MCNPX version 2.1.5, CCC-705, RSICC Computer Code Collection, Oak Ridge National Laboratory (1999).

4) International Commission on Radiological Protection. "Conversion coefficients for used in radiological protection against external radiation". ICRP Publication 74, Ann. ICRP 26(3/4) (Oxford; Pergamon Press) (1996).

5) S. Iwai et al., "Overview of fluence to dose equivalent conversion coefficients for high-energy radiations-calculation methods and results of effective dose equivalent and effective dose per unit particle fluence”, Proc. of SATIF3,, (1997).

6) H. Iwase, K. Niita and T. Nakamura, "Development of GeneralPurpose Particle and Heavy Ion Monte Carlo Code" Nucl. Sci. Technol. 39, No. 11, 1142 (2002).

7) K. Gunzert-Marx, H. Iwase, D. Schardt, and R. S. Simon "Secondary beam fragments produced by $200 \mathrm{MeV} / \mathrm{u}{ }^{12} \mathrm{C}$ ions in water and their dose contributions in carbon ion radiotherapy“, New Journal of Physics 10 (2008).

8) H. Iwase, K. Gunzert-Marx, E. Haettner, D. Schardt et al., "Experimental and theoretical study of the neutron dose produced by carbon ion therapy beams", Radiat. Prot. Dosim., 1, (2007).

9) R. H. Olsher, H. H. Hsu, A. Beverding, et al., "WENDI: an improved neutron rem meter". Health Phys., 79(2), 170 (2000).

10) M. Sasaki E. Kim, T. Nunomiya, T. Nakamura, N. Nakao, T. Shibata, Y. Uwamino, S. Ito, A. Fukumura, "Measurements of High-Energy Neutrons Penetrated Through Concrete Shields Using Self-TOF, NE213, and Activation Detectors" Nucl. Sci. and Engeer., 2, vol. 141, no2, 140 (2002). 